\title{
Geography speaks: performative aspects of geography
}

\author{
C. Schurr \\ Geographisches Institut, Universität Zürich, Winterthurerstr. 190, 8057 Zürich, Switzerland \\ Correspondence to: C. Schurr (carolin.schurr@geo.uzh.ch)
}

Sullivan, R.: Geography Speaks, Ashgate, Farnham, 189 pp., ISBN-13: 9781409420095, E67.99, 2011.

Geography speaks - seit der linguistischen Wende, die in der deutschsprachigen Geographie vor allem durch die Arbeiten von Antje Schlottmann (2005) eingeleitet wurde, wissen wir, dass die Geographie Wirklichkeit durch ihre Karten, Theorien und Sprechakte performativ herstellt. Wer denkt, dass Rob Sullivans Geography Speaks nur längst bekanntes widerkäut, der irrt jedoch. Sullivan entwirft in seinem brillanten und durchweg humorvoll geschriebenen Buch ein umfassendes Programm für eine Performative Geographie, das sowohl auf theoretischer als auch auf empirischer Ebene neue Erkenntnisse für Forschende der Geographie, Wissenschaftssoziologie und -geschichte und Sprachwissenschaften liefert.

Bevor er sich im ersten Teil des Buches an verschiedenen performativen Ansätzen abarbeitet, warnt Sullivan, dass ,this first part of the text may require some patience on the part of the readers, especially those who are strictly interested in geography“ (S. 4). Tatsächlich setzt dieser erste Teil des Buches gewisse Vorkenntnisse des Lesenden voraus, denn Sullivan steigt direkt mit Derridas Kritik an Austins Sprechakttheorie ein, ohne vorher die grundlegenden Prämissen von Austins Denken zu explizieren. Im Vordergrund steht die Frage ob performative Sprechakte nur dann gelingen, wenn sie im tatsächlichen ,realen“ Leben vollzogen werden oder ob sie auch in der Welt der Fiktion wirkmächtig werden können. Anhand Searles Zweifel darüber, dass die „distinctions between [...] fiction and nonfiction [...] all apply more or less" (Searle, 1994:637-638; Hervorhebung durch Searle zitiert, S. 18) zeigt Sullivan, wie unscharf die Grenzen zwischen Fiktion und Realität sind. Vielmehr besteht für ihn eine reziproke Beziehung zwischen den Inszenierungen (performances) des Theaters und der Gesellschaft.

In einem zweiten Schritt diskutiert Sullivan die Rolle des Körpers und des Unterbewusstseins für die Sprechakttheorie. Judith Butlers Verdienst sieht Sullivan in ihrer Theoretisierung des Körpers in der Sprechakttheorie. Der Körper kann nicht ausserhalb des Sprechakts positioniert werden, sondern der organische Körper ist vielmehr Voraussetzung für jeden Sprechakt (Butler, 2007:144). Der Erfolg der performativen Wende bzw. die Tatsache, dass Austins Performanz der Sprechakttheorie glückt (felicitous), hängt für Sullivan eng mit dem Erfolg von Butlers (1990) Buch Gender Trouble zusammen. Butler spricht dem vergeschlechtlichten Körper jeglichen ontologischen Status ab, indem sie seinen performativen Charakter aufzeigt: Geschlecht ist für sie damit nicht mehr als die ständige Wiederholung und Zementierung von körperlichen Zeichen und anderen diskursiven Mitteln.

In der Ritualisierung von Praktiken, die gleichermassen zentral für Austins Sprechakttheorie und Butlers Dekonstruktion von Geschlechtsidentität ist, sieht Sullivan die Verbindung zu den Performance Studies wie sie von Richard Schechner in den 1980er Jahren gegründet wurden. Für Schechner (1998:357-358) ist performance eine ,inclusive category that includes plays, games, sports, performance in everyday life, and ritual“. Laut Sullivan besteht jedoch zwischen den Performance Studies wie sie Schechner versteht und dem Werk von Austin keine direkte Verbindung. Die Ambiguität des Begriffes ,,performance“ - einerseits als Ausführung und Ausübung einer bestimmten Handlung und andererseits als die Vorführung eines eingeübten und ausgearbeiteten (Kunst)Werkes - und die oft synonyme Verwendung des Begriffs Performativität und performance führt oft zu theoretischen Verwirrungen. Grundsätzlich gilt, dass der Begriff der Performativität eine geglückte performance voraussetzt, das heisst, die Ausübung einer Handlung oder eines Sprechakts hat den gewünschten Effekt erzielt. Das Hochzeitspaar ist nach dem obligatorischen Sprechakt des Standesbeamten verheiratet, das Geschlecht des Kindes nach dem Sprechakt des Arztes eindeutig. Sullivans theoretische Ausführungen über die verschiedenen Strömungen performativer Theorien sind durchweg fundiert und auch für performative „Cracks" lesenswert. Schade ist allerdings, dass er nicht weiter auf die Unterschiede zwischen den Begriffen Performativität und performance und deren Verwendung in der 
Geographie eingeht. Eine solche Diskussion hätte es möglich gemacht, die Schwierigkeiten und Potenziale des für die non- oder mehr-als-repräsentationalen Geographien zentralen Begriffs performance näher zu beleuchten (Lorimer, 2005, 2008; Thrift, 2008). Wichtig ist also festzuhalten, dass sich Sullivan in seinem Buch primär auf den Performativitätsbegriff von Austin, Searle, Derrida und Butler bezieht und für ihn die Frage im Vordergrund steht, wie geographische Sprechakte performativ, das heisst in dem Sinne wirkmächtig werden, dass sie eine Realität schaffen, die sie zu beschreiben versuchen.

Im zweiten Kapitel untersucht Sullivan in Anlehnung an die Wissenschaftssoziologie und die Arbeiten von Bruno Latour, wie wissenschaftliche Tatsachen performed, also hergestellt werden. Sullivan interessiert sich hier einerseits für die Art und Weise wie Geographien der Wissensproduktion entstehen, die durch bestimmte politische, gesellschaftliche und ökonomische Kontexte hervorgebracht werden. Andererseits zeigt er anhand der Biographie von David Harvey auf, wie dessen Wissensproduktion durch seine biographische Verortung in einer bestimmten Zeit (Nachkriegszeit und 60er Jahre Revolten) und einem bestimmten geographischen Kontext (Cambridge, Bristol) geprägt wurde.

Im zweiten Teil des Buches Geographical Applications of the Performative wird es dann wie versprochen endlich wirklich ,geographisch“. Sullivan untersucht fünf Aspekte der Geographie durch den Fokus performativer Theorien: Die Performanz von Orten, die Performanz der Kartographie, die performative Trennung zwischen öffentlichem und privatem Raum, die Performanz geodeterministischer Geopolitik und zuletzt die Auto-Performanz der Geographie.

In The Performance of Place diskutiert Sullivan zunächst die Schwierigkeit den Begriff ,,place“ überhaupt zu definieren bevor er anschliessend zahlreiche Beispiele nennt, wie Orte performed werden. Lieder wie „New York, New York“ sind zur Synekdoche für die Stadt geworden: New York ist die Stadt der Städte und damit ein Synonym für die Stadt selbst. Märchen, Erzählungen und alltäglicher gossip sind weitere performances, die Orte hervorbringen. Der performative Aspekt geographischer Verortung wird durch Sullivans Diskussion über die Macht der (kolonialen) Ortsbenennung noch deutlicher. Christopher Kolumbus Sprechakt der „Neuen Welt“ ist hier exemplarisch dafür wie WeltOrdnungen und damit geopolitische Machtverhältnisse über Sprechakte hergestellt werden.

Unter dem Titel The Performance of Cartography setzt sich Sullivan im vierten Kapitel mit dem ,,performativen vehicle“ (S. 83) der Disziplin auseinander: der Karte. Spannend ist hier seine Diskussion der - im Sinne von Austin - unglücklichen (infelicitous) Geschichten der Kartographie wie zum Beispiel der Versuch der USA, den Meridian durch Philadelphia verlaufen zu lassen oder ähnliche Initiativen für einen Pariser oder Berliner Null Meridian. Des Weiteren geht er auf die selbstlegitimierende Rolle von Karten in der kolonialen Geschichte ein und diskutiert anhand der nationalso- zialistischen Geschichte Deutschlands die politische Instrumentalisierung von Karten.

Kapitel 5 beschäftigt sich mit der „Performance of Private and Public Space“. Die performative Markierung von Räumen als privat, öffentlich, öffentlich-privat und privatöffentlich in kapitalistischen Gesellschaften ist ein wichtiges Thema in verschiedenen Teildisziplinen der Geographie. Sullivan zeigt durch eine Reihe von empirischen Beispielen auf, wie die Abgrenzungen zwischen diesen vier unterschiedlichen Typen von Raum durch performative Sprechakte vollzogen werden.

Der performative geographische Höhepunkt wird in Kapitel 6 erreicht. Kaplan's The Revenge of Geography zeigt wie kaum ein anderes Beispiel die performative Macht eines geodeterministischen Diskurses. „People and ideas influence events, but geography largely determines them“ (Kaplan, 2009:1) - Sullivan reagiert humorvoll auf Kaplans Ignoranz geographischer Fachdebatten: „This is stated with all earnestness and complete sincerity in an era when others have proclaimed the death of distance, the placelessness of place, and advocated the idea that globalization has rendered geography nugatory“ (S. 123). Facettenreich und aus historischer Perspektive zeigt Sullivan in diesem Kapitel auf, wie performative Sprechakte von politischen Führern (und dem Führer insbesondere) benutzt wurden, um geopolitische Veränderungen hervorzurufen. Interessant ist hier insbesondere seine Abhandlung über die Rolle, die Geographen wie Friedrich Ratzel und Karl Haushofer in der erfolgreichen Inszenierung (performance) dieser Politik gespielt haben.

Das letzte Kapitel beschäftigt sich mit der „AutoPerformance of Geography“. Am Beispiel von Lefebvres Right to the City, Harveys Social Justice and the City und Yi-Fu Tuans Humanistic Geography geht der Autor der Frage nach wie diese drei Geographen (leider werden hier tatsächlich ausschliesslich nur die Werke von männlichen Geographen untersucht) versucht haben, die Disziplin durch ihre definitorischen Performative zu disziplinieren. Sullivan betont am Ende der Diskussion, dass die performances dieser drei Geographen durchweg geglückt sind, was sich an dem langfristigen Effekt ihrer Arbeiten auf theoretische und empirische Debatten in der Geographie zeigt.

In seinem Schlusswort betont Sullivan noch einmal, dass seine Auslegeordnung der performativen Aspekte der Geographie durch die Brille von Austins Sprechakttheorie selbst eine Performanz ist, die so noch nie aufgeführt worden ist. Er hofft, dass durch seine Performanz deutlich geworden ist, dass die Geographie performed - egal ob in positiver oder negativer Art und Weise wie im Falle des Geodeterminismus. Ziel des Buches sei es zu zeigen, dass ,geography matters". Sullivans zentrale performative Forderung, die er in dem Buch ausformuliert, besteht darin zu zeigen, dass die Geographie eine wichtige Rolle in der Wissenschaft und Gesellschaft spielt. Denn, ,geography is performing, everywhere" (S. 174). 
Geography Speaks ist tatsächlich ein beeindruckendes Werk, das eine ausserordentliche theoretische Komplexität mit einer ungeheuren empirischen Dichte verbindet. Gleichzeitig wirft das Buch viele Fragen auf und bringt selbst eine Karte performativer Geographien hervor, die durchaus weisse Flecken aufweist. Überraschend wenig beschäftigt sich Sullivan mit den Arbeiten in der Geographie, die explizit eine performative Perspektive aufgegriffen haben und eine solche für geographische Forschung fruchtbar gemacht haben. Weder Gregson und Roses (2000) einschlägiger Artikel Taking Butler elsewhere: performativities, spatialities and subjectivities noch Arbeiten aus der feministischen Geographie, die Butlers Konzept der Performativität als erste in die Geographie eingeführt haben (s. zum Beispiel Bell et al., 1994; McDowell und Court, 1994; Nelson, 1999), finden in Geography speaks Beachtung. Im Sinne des performativen Charakters disziplinärer Wissenschaftsgeschichte(n) können solche „cartographic silences“ (S. 90) im Falle einer erfolgreichen Performanz von Sullivans Inszenierung durchaus problematisch sein. Sympathisch ist, dass er mehrfach betont, dass er nicht für sich beansprucht, alle performativen Aspekte der Geographie beleuchtet zu haben und auch anerkennt, dass er nicht der erste ist, der sich mit diesen beschäftigt hat. Trotz der weissen Flecken ist Sullivans Werk äusserst lesenswert, nicht nur weil es spannende Einblicke in die Wissenschaftsgeschichte der Disziplin und andere geographischen Fragen bietet, sondern auch weil es eloquent und äusserst humorvoll geschrieben ist.

\section{Literatur}

Bell, D., Binnie, J., Cream, J., und Valentine, G.: All hyped up and no place to go, Gender Place Cult., 1, 31-47, 1994.

Butler, J.: Gender trouble: feminism and the subversion of identity, New York, Routledge, 1990.

Butler, J.: Afterword, in: The scandal of the speaking body, Herausgeber: Sun, E., Peretz, E., und Baer, U., New York, 2007.

Gregson, N. und Rose, G.: Taking Butler elsewhere: performativities, spatialities and subjectivities, Environ. Plann. D., 18, 433452, 2000.

Kaplan, R. D.: The revenge of geography, Foreign Policy, 96-105, 2009.

Lorimer, H.: Cultural geography: the busyness of being "more-thanrepresentational”, Prog. Hum. Geogr., 29, 83-94, 2005.

Lorimer, H.: Cultural geography: non-representational conditions and concerns, Prog. Hum. Geogr., 32, 551-559, 2008.

McDowell, L. und Court, G.: Performing work: bodily representations in merchant banks, Environ. Plann. D, 12, 727-750, 1994.

Nelson, D. M.: A finger in the wound. Body politics in Quincentennial Guatemala, Berkeley, University of California Press, 1999.

Schlottmann, A.: RaumSprache, Ost-West-Differenzen in der Berichterstattung zur deutschen Einheit, München, Franz Steiner Verlag, 2005.

Thrift, N.: Non-representational theory: space, politics, affect, London, Routledge, 2008. 